\title{
Ärzte der Seele
}

Médecins Sans Frontières s'engage pour la fourniture des soins en zones de crise. Human Rights Orchestra s'investit pour la promotion des droits de l'Homme. Tous les deux collaborent pour la première fois dans le cadre du concert de charité organisé à l'occasion du Forum international des droits de l'Homme à Lucerne (IHRF).

Die Menschen in der arabischen Welt sprechen von gewonnener Freiheit. Obschon ihre Zukunft mehr als ungewiss ist. Aber ihre Massenproteste haben Wirkung gezeigt. Von Tunesien über Libyen bis nach Ägypten. Die Rufe nach Rechten verhallen nicht ungehört. Es ist ein grenzübergreifendes Pochen auf die Durchsetzung von bisher vorenthaltenen Menschenrechten. Recht auf freie Meinungsäusserung, Recht zum freien Protest, Recht auf freie Wahlen. libyschen Bürgerkrieges und der Umwälzungen im Orient mit medizinischem Material zu beliefern. «Wir sind äusserst besorgt über den verwehrten Zugang zu medizinischer Versorgung und den Zustand der Patienten in öffentlichen Gesundheitseinrichtungen in von der Regierung kontrollierten Gebieten», sagt Bruno Jochum, Operations-Direktor von Médecins Sans Frontières in einer kürzlich veröffentlichten Medienmitteilung bezogen auf Libyen.

\section{«Das Human Rights Orchestra spielt klassische Musik mit der Mission, die Menschenrechte zu fördern, und schaut damit über den musikalischen Tellerrand hinaus.»}

\section{Festgehalten an der Grenze}

Hilfeleistungen für die aufständischen Völker kommen bisher kaum von den Politikern in Berlin, Paris oder London, vielmehr von humanitären Organisationen und engagierten privaten Personen. Seit Wochen versuchen auch Médecins Sans Frontières (Ärzte ohne Grenzen), Verwundete und Hilfsbedürftige des
Seit Wochen stehen Ärzte, Apotheker und Krankenschwestern an der Landesgrenze, wo ihnen der Zutritt zu den Kampfgebieten absichtlich verweigert wird. Ein nervenaufreibendes und gefährliches Geduldsspiel. «Dabei ist es zwingend notwendig, dass die Menschen in den Kampfgebieten die Möglichkeit haben zu flüchten, um sichere Orte aufzusuchen - so-
Korrespondenz: Gregor Erismann International Human Rights Forum Lucerne (IHRF) PHZ Luzern

Bellerivestrasse 19 CH-6006 Luzern Tel. 0412284730

gregor.erismann@ihrf.phz.ch

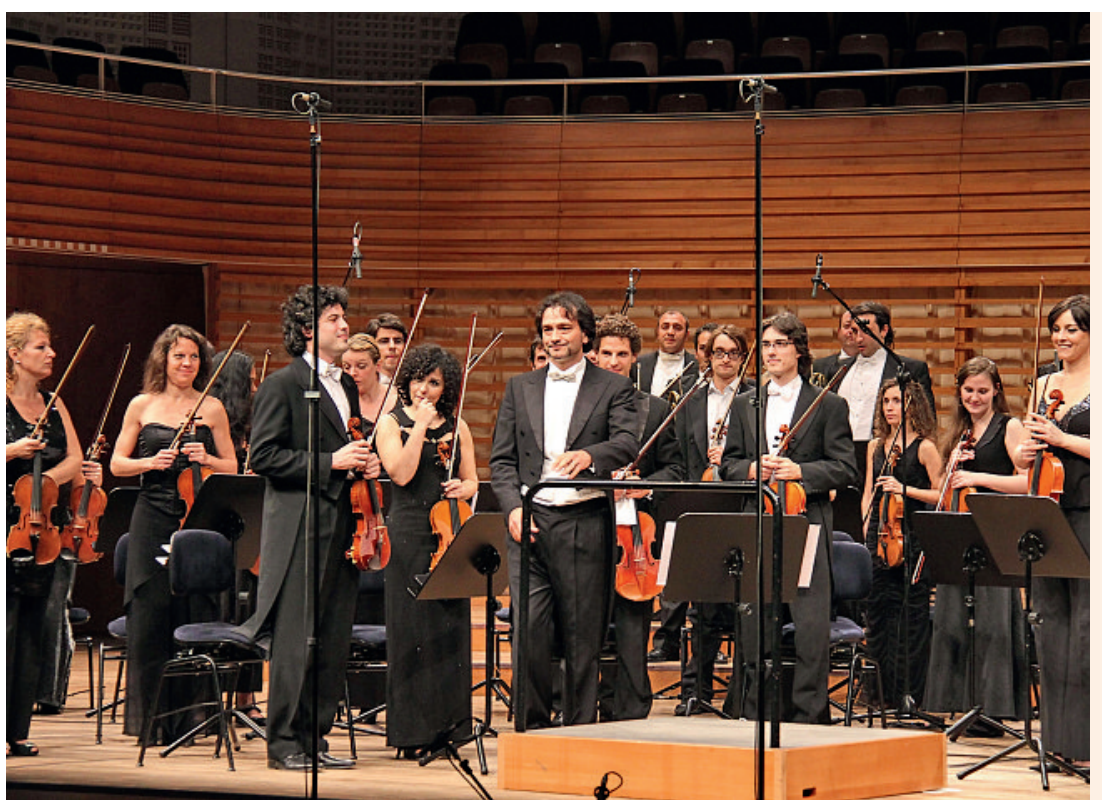

Das Human Rights Orchestra mit seinem Chefdirigenten Alessio Allegrini wird zusammen mit der Pianistin Hélène Grimaud zugunsten von Médecins Sans Frontières spielen. 
wohl innerhalb wie auch ausserhalb Libyens», sagt Jochum weiter. 22 Tonnen an medizinischen Hilfsgütern wurden seit dem 24. Februar (Stand: 18. März 2011) nach Libyen geliefert. 11 weitere Tonnen stehen bereit. Der Bedarf an medizinischen Gütern konnte bisher im unübersichtlichen Kampf zwischen Oppositions- und Regierungstruppen nur ungenügend gestillt werden. Das Engagement von Médecins Sans Frontières hält an.

\section{Ein Megafon für die Menschenrechte}

Während sich Médecins Sans Frontières weltweit um die Stabilisierung und Verbesserung der Lebensbedingungen von Menschen in Not bemüht, ist die Organisation auf finanzielle Unterstützung angewiesen, die grösstenteils von privaten Spendern stammt. Zur Unterstützung der humanitären Arbeit findet am 24. Mai 2011 ein Benefizkonzert im Rahmen des 8. Internationalen Menschenrechtsforums Luzern (IHRF) statt. Das Human Rights Orchestra spielt im KKL Luzern zugunsten von Médecins Sans Frontières. 2009 vom italienischen Starhornisten und Dirigenten Alessio Allegrini gegründet, setzt sich das Orchester aus namhaften Musikern verschiedener grosser Orchester der ganzen Welt zusammen. Dabei sind Musiker der Berliner Philharmoniker, der Wiener Philharmoniker oder der Orchester der Opernhäuser La Scala in Mailand und La Fenice in Venedig. Sie alle engagieren sich ehrenamtlich für das Projekt. «Musik lehrt die Leute zuzuhören. Wir sind ein Megafon, welches die Botschaft der Menschenrechte so weit wie möglich verbreiten will», sagt der Gründer und Dirigent Alessio Allegrini.

\section{Das Zusammenspiel der Ärzte}

Das Human Rights Orchestra spielt klassische Musik mit der Mission, die Menschenrechte zu fördern, und schaut damit über den musikalischen Tellerrand hinaus. «Musik ist nicht bloss die exakte Wiedergabe von Noten und Intonation. Musik öffnet das Herz und versprüht positive Energie, sie verbindet Menschen und durchdringt die Stille», so Allegrini. Die Konzerte des Human Rights Orchestra sind Kommunikationsmittel, um auf Menschenrechtsverletzungen und menschenunwürdige Lebenssituationen aufmerksam zu machen - wie in Libyen oder im Sudan; die Liste der Länder ist lang. «Die einen retten Menschenleben und die anderen wollen den Geist der Menschen mit ihrer Musik berühren, um auf die Einhaltung der Menschenrechte aufmerksam zu machen. Das passt doch zusammen», so Allegrini. Mit Begeisterung habe man im Orchester darauf reagiert, am 24. Mai im KKL Luzern für Médicins Sans Frontières zu spielen. Kein Zufall, dass sich das Orchester zur Gründungszeit «Musiker ohne Grenzen» nennen wollte, den Namen aber wieder abgab, da er bereits verwendet wurde.

Hélène Grimaud wird in Luzern als Solistin auf der Bühne stehen. Auch sie aus Engagement für die Sache, ohne Gage. Die Pianovirtuosin gehört seit Jahren zu den gefeierten Stars auf den Klassikbühnen dieser Welt und ist regelmässiger Gast beim Lucerne Festival.

In Luzern ebenfalls auftreten wird die italienische Cellistin Sara Gentile, ein festes Mitglied des Human Rights Orchestra und von dessen positiver Energie überzeugt: «Seit ich beim Human Rights Orchestra dabei bin, habe ich das Gefühl «nützlich〉 zu sein. Meine Musik kann etwas verändern», sagt sie. Die Italienerin engagierte sich zuvor in der Politik, dort aber wurde zu viel diskutiert und zu wenig gehandelt. «Jetzt habe ich das Gefühl, ein Teil von etwas Bedeutendem zu sein, wir machen mit unserer Musik und unseren Benefizkonzerten einen Unterschied in der Welt.» Durch ihre Musik sollen all jene eine Stimme erhalten, die sonst nicht gehört werden.

Im Rahmen des 8. Internationalen Menschenrechtsforums Luzern (IHRF):

\section{IHRF Concert Classic 2011 zugunsten Médecins Sans Frontières}

24. Mai 2011 KKL Luzern - 19.30 Uhr:

Human Rights Orchestra, Leitung Alessio Allegrini

Hélène Grimaud, Klavier

Zoltán Kodály - Tänze aus Galanta

Maurice Ravel - Klavierkonzert in G-Dur

György Ligeti - Concert Românesc

Antonín Dvořák - 9. Symphonie in e-Moll op. 95 (Aus der Neuen Welt)

Konzerteinführung mit Andreas Müller-Crepon (Musikredaktor DRS 2)

Kartenvorverkauf: www.kkl-luzern.ch oder 0412267777

Preise: 175 CHF / 140 CHF / 110 CHF / 80 CHF / 50 CHF

Weitere Informationen zum Konzert und zu den Hospitality-Möglichkeiten: www.humanrightsforum.ch

Zum Human Rights Orchestra: www.musiciansforhumanrights.org

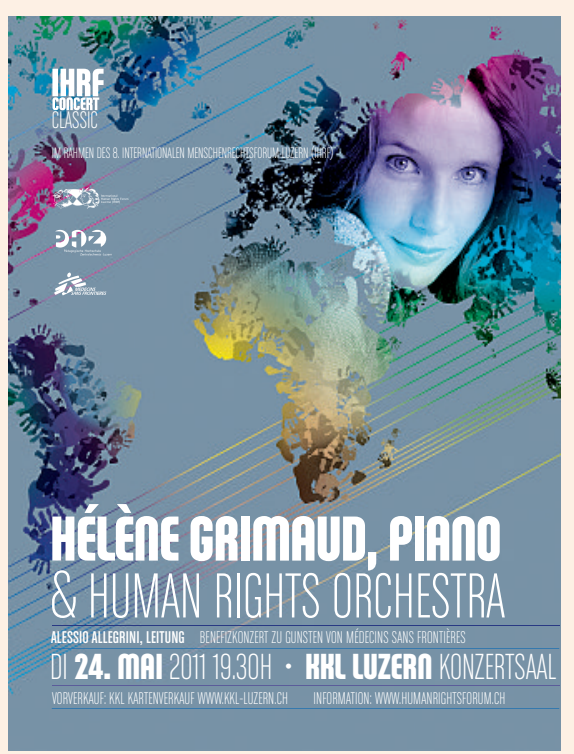

